\title{
The Feasibility of Applying Inferior Vena Cava Collapse Index to Guide the Use of Fluid Therapy in Neonates with Pulmonary Hemorrhage on Mechanical Ventilation
}

HongJuan JIN ( $\square$ georgechan_fjmu@163.com )

Fudan University Zhongshan Hospital Xiamen Branch https://orcid.org/0000-0001-6028-8243

Xiaoyan LIU

Children's Hospital of Fudan University Xiamen Branch

Ying HUANG

Children's Hospital of Fudan University Xiamen Branch

\section{Research}

Keywords: neonates, IVC-Cl, fluid therapy

Posted Date: October 29th, 2021

DOI: https://doi.org/10.21203/rs.3.rs-1002197/v1

License: (c) (i) This work is licensed under a Creative Commons Attribution 4.0 International License.

Read Full License 


\section{Abstract}

Purpose. Explore the feasibility of applying Inferior Vena Cava Collapse Index (IVC-Cl) in guiding the use of fluid therapy in neonates with Pulmonary Hemorrhage (PH) on mechanical ventilation. Construct a novel and non-invasive technique in accurately and dynamically assess the fluid volume of neonates.

Methods. Cases met the diagnostical criteria of Pulmonary Hemorrhage (PH) admitted between July 2016 to June 2018 were reviewed and included into the Control Group for this study. Cases met the same said criteria between July 2018 and June 2020 were included into the Experimental Group. PHILIPS Bedside color Doppler ultrasound system was used in detecting and calculating the IVC-Cl, which was later used for patients in experimental group to guide the practice of fluid therapy. A total of 54 cases were reviewed with 8 cases excluded for death during hospitalization. 24 cases were included into the control group, 22 cases were included into the experimental group.

Results. Fluid intake volumes were recorded at $0-6^{\text {th }}$ hours, $6^{\text {th }}-24^{\text {th }}$ hours and $24^{\text {th }}-48^{\text {th }}$ hours with the onset of $\mathrm{PH}$ as the initial observation point. For 22 patients in experimental group, the volumes were respectively $(64 \pm 6) \mathrm{ml} / \mathrm{Kg},(71 \pm 8) \mathrm{ml} / \mathrm{Kg}$ and $(102 \pm 10) \mathrm{ml} / \mathrm{Kg}$. In the control group, the volumes were $(60 \pm 4) \mathrm{ml} / \mathrm{kg},(93 \pm 4) \mathrm{ml} / \mathrm{kg}$ and $(105 \pm 9) \mathrm{ml} / \mathrm{kg}$, respectively. The $P$ values of the two groups during the same time period were $0.013,0.000,0.361$, suggesting the differences of fluid intake between the two groups during the first 6 hours and the following 18 hours after the onset of $\mathrm{PH}$ were of statistical significance.

The time taken for the improvement of lactate concentration and oxygenation index for the experimental group were (26.4 \pm 6.7$) \mathrm{h}$ and $(22.6 \pm 5.8) \mathrm{h}$, respectively. The time taken for the same two indexes to improve in the control group were $(38.5 \pm 6.7) \mathrm{h}$ and $(27.0 \pm 5.0) \mathrm{h}$. $\mathrm{P}$ values for these two indexes between two groups were 0.015 and 0.009 , suggesting statistically significant differences.

Conclusion. Ultrasound guided IVC-CI measurement can dynamically evaluate volume status, guide fluid therapy in a targeted manner. A more responsive and selective fluid therapy achieved this way can alleviate the patients' conditions quicker than traditional therapeutic approaches.

\section{What Is Known}

- Ultrasound guided IVC-Cl assessment and using it to evaluate patient's volume status is quite common in adult patients.

- IVC-Cl can provide timely and dynamic readings of the patient's volume status.

- There's a lack of applicable non-invasive technique to assess an infants \& neonates volume status.

\section{What is New}

- This article introduces the said evaluation index in neonatal ward to guide the fluid therapy and yielded satisfying results, suggesting the use of IVC-CI in neonates can better guide the use of fluid 
therapy.

\section{Introduction}

Pulmonary hemorrhage in neonates is a massive hemorrhage involving at least 2 lobes of the lungs, resulting in high morbidity and mortality rate. The particularly high incidence of pulmonary hemorrhage in neonates can be ascribed to their anatomical and developmental characteristics ${ }^{[1]}$. Timely mechanical ventilation and hemostatic treatment play a decisive role, while fluid therapy is also particularly critical. Reasonable and effective fluid therapy can provide the body with a stable internal environment, reduce further tissue injury, alter the course of the disease and produce more favorable outcome ${ }^{[2]}$.

Perspective approaches were applied in conducting this study. Routine and conventional treatments including mechanical ventilation and hemostasis were performed while calculating the Inferior Vena Cava Collapse Index (IVC-CI) [(Maximum diameter of I.VC - Minimum diameter of I.VC)/Maximum diameter of I.VC] ${ }^{[3][4]}$ to assess the effectiveness of fluid therapy.

\section{Patients And Methods}

2.1 Case Collection. This study was conducted at the Neonatal Intensive Care Unit (NICU) of Children's Hospital of Fudan University Xiamen Branch (Xiamen Children's Hospital), one of the regional's medical centers for neonatal diseases. Cases met the diagnostical criteria of Pulmonary Hemorrhage (PH) ${ }^{[5][6]}$ admitted between July 2016 to June 2018 were reviewed and included into the Control Group for this study. Cases met the same said criteria between July 2018 and June 2020 were reviewed and included into the Experimental Group. Exclusion criteria included: 1. PH occurred more than 1 week after birth; 2. Patients with severe liver failure, idiopathic pulmonary hemosiderosis, intracranial hemorrhage; 3. congenital fatal malformations, hypoplasia of the trachea or chest/abdominal wall, severe congenital heart diseases, hemophilia and other coagulation abnormalities; 4. patients dies during hospitalization. PHILIPS bedside color Doppler ultrasound system was used in detecting and calculating the IVC-Cl, which was later used for patients in experimental group to guide the practice of fluid therapy. A total of 54 cases were reviewed with 8 cases excluded for death during hospitalization. 24 cases were included into the control group, 22 cases were included into the experimental group.

This study was approved by the Ethics Committee of the Children's Hospital of Fudan University Xiamen Branch (Xiamen Children's Hospital) and was performed in compliance with the Declaration of Helsinki. Written consent form from all patients' legal guardians were received.

2.2 Methods. Bedside ultrasound system was used to assess the IVC-Cl of patients in experimental group to evaluate the effectiveness of fluid therapy. Ultrasound assessment was performed by the same group of experienced physicians. 
2.2.1 Ultrasound Data Collection. The patients were placed supine and sedated, ultrasound probe was placed to the right of the subxiphoid process to show the long-axis view of the inferior vena cava. 0.5-1 $\mathrm{cm}$ below where the hepatic vein meets the inferior vena cava was selected as the measurement point.

2.2.2 Therapeutic Approaches. All cases included into the study were on mechanical ventilation support, most of the patients were clinically diagnosed basing on the observation of intratracheal hemorrhage. Immediately after the onset of $\mathrm{PH}$, the IVC-CI was acquired with the said method. Therapeutic principles include correction of acidosis and plasma transfusion. IVC-CI was assessed again both before and after the transfusion. Considering there were no commonly acknowledged practice of using IVC-CI to guide fluid therapy in neonates, we referred to the guidelines published for adults, which indicate for limited fluid intake when IVC-CI is below $15 \%$ and rather aggressive fluid therapy when IVCC reached above $50 \%$. Once the active hemorrhage stopped and vitals including blood oxygen saturation, blood pressure, heart rate stabilized, IVC-Cl was monitored every 4 hours with the observation cut-off point at 72 hours after the onset of $\mathrm{PH}$.

2.2.3 Observational Index. $\mathrm{pH}$, partial pressure of carbon dioxide $\left(\mathrm{pCO}_{2}\right)$, partial pressure of arterial oxygen $\left(\mathrm{pO}_{2}\right)$, blood lactate concentration, oxygenation index $\left[\left(\mathrm{FiO}_{2} \times\right.\right.$ Mean Arterial Pressure $\left.) / \mathrm{PaO}_{2}\right]$, and heart rate and the time intervals for the improvement of said indexes were recorded. The correlation of IVC-Cl and changes of mean plasma hemoglobin concentration $(\mathrm{Hb})$ was monitored. The fluid intake of the patients during the observation period was recorded.

2.2.4 Statistical Analysis. Measurement data were expressed in mean \pm standard manners $(\mathrm{x} \pm \mathrm{s})$, and ttest was used for comparison between groups, and categorical data were presented as number of cases or ratio, and chi-square test was used for comparison between groups. All tests of statistical hypotheses were two-sided, and $p \leq 0.05$ was considered statistically significant. All data were analyzed using SPSS 22.0 (SPSS Inc., Chicago, IL, USA).

\section{Results}

3.1 Demographic Data. All 54 cases were included into the study, 8 cases were excluded because of death during hospitalization. 22 cases were included into the experimental group and 24 cases in control group. Basic demographic data included gestational ages, birth weight, gender, time of the onset of PH. The differences were not statistically significant. Basic demographic data was compiled in the Table 1.

3.2 Major Arterial Blood Gas Index at the Onset of PH. Indexes included pH, partial pressure of carbon dioxide $\left(\mathrm{pCO}_{2}\right)$, partial pressure of arterial oxygen $\left(\mathrm{pO}_{2}\right)$, blood lactate concentration, oxygenation index (OI). Heart rate as an important vital was also included for analysis. Data in Table 2 suggested the differences were of no statistical significance.

3.3 Correlation between IVC-Cl and changes of hemoglobin concentration. 
In clinical practice, hemoglobin concentration is used widely to assess whether or not there is an active bleeding. This index usually drops when internal hemorrhaging is underway. To establish that IVC-Cl can reflect on the changes of fluid volume, the baseline hemoglobin concentration $\left(\mathrm{Hb}_{B}\right)$ and the concentration $\left(\mathrm{Hb}_{\mathrm{H}}\right)$ after diagnosed with $\mathrm{PH}$ were recorded. As indicated in Table 3 and Figure 1 , the greater changes hemoglobin concentration $\mathrm{Hb}$ exhibited, the greater IVC-Cl elevated, suggesting a strong correlation between these two indexes, also proving that IVC-Cl can dynamically reflect on the body's fluid volume status.

3.4 As demonstrated in Table 4, a comparison between the fluid intake of 2 groups was drawn, the differences of intake fluid volume in the first 6 hours and the 6th to 24th hours after the onset of PH were of statistical significance, while the intake fluid volume between the 24th to the 48th hours after the onset of $\mathrm{PH}$ was considered statistically insignificant.

3.5 During the pathophysiological process of $\mathrm{Hb}$, blood lactate concentration is elevated because of the cellular anaerobic respiration process. Oxygenation Index $\left[(\mathrm{FiO} 2 \times \mathrm{MAP}) / \mathrm{PaO}_{2}\right]$ is also significantly elevated ${ }^{[7]}$. Hence, there two indexes can reflect on the body's circulatory and respiratory conditions. During the observation period, the time interval took for blood lactate concentration to drop back to normal range were recorded ( $\left.\mathrm{T}_{\text {Lactate }}\right)$ and compared between different groups. A lactate concentration below $4 \mathrm{mg} / \mathrm{dl}$ was considered normal. The period of time took for Oxygenation Index (P/F) to drop by $50 \%$ was also recorded $\left(T_{0 I}\right)$. Compiled data in Table 5 suggested that the differences between two groups were of statistical significance.

\section{Discussion}

Pulmonary hemorrhage in neonates is mostly hemorrhagic, the pathophysiological changes this time is mostly about increase vascular filtration pressure, increased ventricular preload ${ }^{[5]}$. Hemorrhaging thus leads to hypovolemia, and if there is a lack of relatively aggressive fluid resuscitation during this period and an untimely response to hypovolemia, dramatic circulatory volume fluctuations and inadequate perfusion of vital organs such as the heart and brain may lead to rapid progression of the conditions ${ }^{[8]}$. Empirical and stereotyped correction of acidosis and transfusion may cause fluid overload. Increased secretion of Endothelin-1 (ET-1) of the vascular endothelial cells is triggered in response to PH. Contraction of smooth muscle and aggravated pulmonary edema can result in elevated pulmonary arterial pressure. Hypoxemia resulted from PH may cause hypoxic myocardial injury and cardiac dysfunction. All these factors lead to increased circulatory burden, causing tissue edema, organ dysfunctions and circulatory failure. Consequently, relatively limited fluid intake to alleviate the circulatory burden and thus reverse the process of pulmonary edema may avoid the development of cardiac failure, eventually reduces mortality ${ }^{[9]}$.

Therefore, the introduction of volume assessment to guide fluid therapy in neonatal PH is crucial. In contrast, preterm infants are inherently characterized by unclosed ductus arteriosus, unclosed foramen 
ovale, immature lung development, relative pulmonary hypertension, right ventricular predominance, and unstable circulatory status ${ }^{[1]}$. Moreover, indicators more frequently used in clinical practice such as heart rate, blood pressure, urine volume, and lactate concentration are easily affected by many factors such as stimulation, body temperature and respiration, making it difficult to assess volume status accurately. In order to obtain dynamic indicators such as central venous pressure, pulmonary capillary wedge pressure, per beat variability and pulse pressure variability, invasive operations are required with complications associated with infection, thrombosis, hematoma, pneumothorax, and operation failure, making it even more difficult to obtain in preterm infants.

Recent years' clinical practice and scientific research revealed the effectiveness of applying IVC-CI in dynamically assessing the volume status, making it a comprehensive evaluation index of cardiac functional status. Because of the fact that IVC-Cl can be acquired in a non-invasive manner, repeated assessment can be performed to evaluate the effectiveness of fluid therapy ${ }^{[10]}$. Zhu Weihua et al. found that compared with patients breathing spontaneously, IVC-Cl can more accurately evaluate fluid load and fluid responsiveness in patients on mechanical ventilation, producing timely report on the effectiveness of the therapeutic approaches ${ }^{[11]}$. The fast respiratory rate and the short distance from the inferior vena cava to the atrium in preterm infants make accurate measurement of IVC-CI relatively difficult. In contrast, children with pulmonary hemorrhage are critically ill and poorly responsive, or are passively ventilatordependent due to sedation, which is suitable for the application of IVC-CI to assess volume status. In this study, hypoxia, hypercapnia, elevated lactate concentration, and increased heart rate were found in all patients with $\mathrm{PH}$, with no significant difference between the two groups. The inferior vena cava collapse index was found to be between $50 \%$ and $75 \%$ in the early stage of $\mathrm{PH}$ in 22 patients in the experimental group, and Figure 1 reflects a positive correlation between the IVC-Cl and the degree of hemoglobin concentration decline in the early stage of $\mathrm{PH}$. Similar to the findings of E Wilkman et al. who reported that IVC-CI can accurately assess volume status at positive end-expiratory pressure ventilation mode ${ }^{[12]}$.

The study on the relation between IVC-Cl and fluid status is relatively scarce. A foreign study with crosssectional analysis of neonates found that IVC-Cl correlated well with central venous pressure, but not with either gestational age or birth weight. Evidently, IVC-CI was not influenced by birth weight or gestational age ${ }^{[13]}$. The results of this study were similar to those of adult studies in that the IVC-Cl correlated well with CVP [14] [15].

In the experimental group of this study, patient's urine volume, heart rate, blood pressure, capillary filling time, lactate, and left ventricular ejection fraction (LVEF) were taken into overall evaluation, while referring to the IVC-Cl practices in adult patient in making therapeutic attempts (Limited fluid resuscitation when IVC-Cl $<15 \%$, active fluid resuscitation when IVC-CI $>50 \%$ ) ${ }^{[16] ~[17] . ~ O u r ~ s t u d y ~ r e v e a l e d ~ t h a t ~ I V C-C l ~ u s u a l l y ~}$ drop by $10-15 \% 6$ hours after the onset of $\mathrm{PH}$. This result was thus used to limit the fluid intake of patients in experimental group. We retrospectively reviewed the cases in control group and discovered that the volume intake in the first 6 hours and between the 6th to 24th hour after the onset of PH was $60 \pm 4 \mathrm{ml} / \mathrm{Kg}$ and $93 \pm 4 \mathrm{ml} / \mathrm{Kg}$ respectively, suggesting the experimental group received more fluid during 
the first 6 hours than the control group, fewer fluid in the following 18 hours, both with statistical significance. The following day witnessed no particular difference in terms of fluid intake. When comparing the time taken for improvement of lactate concentration and oxygenation index in both groups, the experimental group was shorter than the control group, and the difference was significant.

This study showed that ultrasound-guided measurement of IVC-Cl can promptly assess the volume status, provide targeted guidance for fluid therapy, shorten the improvement time of lactate concentration and oxygenation index, and facilitate the recovery of the disease. It is similar to the findings of Clive $\mathrm{N}$ May et al ${ }^{[18]}$ who reported that ultrasound-guided fluid therapy shortened the duration of ICU stay and the duration of mechanical ventilation in patients with severe sepsis.

It is worth noticing that we selected a different ultrasound probing point for measuring the IVC-Cl compared to adult patients. A routine measuring point is in between the confluence of the hepatic vein into the inferior vena cava and the confluence inferior vena cava into the right atrium ${ }^{[19]}$. while we select 0.5-1 $\mathrm{cm}$ below where the hepatic vein meets the inferior vena cava was selected as the measurement point. This alteration was made because of the anatomical short distance between the confluence of hepatic vein into the inferior vena cava and the confluence of inferior vena cava into the right atrium in neonates, and the routine measuring point is subject to the influence of heart beat, consequently affecting the accuracy of the measurement. Moreover, the measurement should not be performed when patients experience respiratory distress, which may lead to an abnormally elevated IVC-Cl, thus causing misdiagnosis and unnecessary treatment.

Due to the small number of cases included into the study and an untraditional measurement point of the IVC-Cl, there may be certain limitations about the conclusion of the study. Hopefully such limitation can be alleviated through future studies which include more samples, leading to a more robust, timely and accurate assessment of the effectiveness of fluid therapy in neonates.

\section{List Of Abbreviations}

IVC-Cl: Inferior Vena Cava Collapse Index

PH: Pulmonary Hypertension

pC02: partial pressure of carbon dioxide

p02: partial pressure of arterial oxygen

Ol: oxygenation index

\section{Declarations}

Funding: Not Applicable. 
Conflicts of interest/Competing interests: All authors declare no conflict of interests.

Availability of data and material: Data available upon request.

Code availability: Not Applicable.

Authors' contributions: JIN H. designed and carried out the study, HUANG Y and LIU X assisted in the study, JIN H. HUANG Y. LIU X. composed and reviewed the article.

Ethics approval: This study received approval from the Ethics Committee of Xiamen Children's Hospital.

Consent to participate: All patient's legal guardians acknowledged and approved the participation of this study.

Consent for publication: Not Applicable.

\section{References}

1. Smith LJ, McKay KO, van Asperen PP, Selvadurai H, Fitzgerald DA. Normal development of the lung and premature birth. Paediatr Respir Rev. 2010;11(3):135-42. doi:10.1016/j.prrv.2009.12.006.

2. Welsh SK, Casey AM, Fishman MP. Pulmonary hemorrhage in infancy: A 10-year single-center experience. Pediatr Pulmonol. 2018;53(11):1559-64. doi:10.1002/ppul.24142.

3. Jassim HM, Naushad VA, Khatib MY, et al. IJV collapsibility index vs IVC collapsibility index by point of care ultrasound for estimation of CVP: a comparative study with direct estimation of CVP. Open Access Emerg Med. 2019;11:65-75. doi:10.2147/OAEM.S176175. Published 2019 Apr 3.

4. Orso D, Paoli I, Piani T, Cilenti FL, Cristiani L, Guglielmo N. Accuracy of Ultrasonographic Measurements of Inferior Vena Cava to Determine Fluid Responsiveness: A Systematic Review and Meta-Analysis. J Intensive Care Med. 2020;35(4):354-63. doi:10.1177/0885066617752308.

5. Neonatology Group of Pediatrics Branch of Chinese Medical Association. Diagnostic and Therapeutic Approaches for Neonates Pulmonary Hemorrhage [J]. Chinese Journal of Practical Pediatrics,2001,16(10):631. DOI:10.3969/j.issn.1005-2224.2001.10.022.

6. Coffin CM, Schechtman K, Cole FS, Dehner LP. Neonatal and infantile pulmonary hemorrhage: an autopsy study with clinical correlation. Pediatr Pathol. 1993;13(5):583-9. doi:10.3109/15513819309048246.

7. Shee B, Anjum F, Rockoff BI. Pulmonary Hemorrhage. In: StatPearls. Treasure Island (FL): StatPearls Publishing; June 4, 2021.

8. SU, BH,LIN HY,HUANG, FK,et al.Circulatory Management Focusing on Preventing Intraventricular Hemorrhage and Pulmonary Hemorrhage in Preterm Infants[J]. Pediatr Neonatol,2016,57(6):453462.

9. Lee M, Wu K, Yu A, et al. Pulmonary hemorrhage in neonatal respiratory distress syndrome: Radiographic evolution, course, complications and long-term clinical outcomes. J Neonatal Perinatal 
Med. 2019;12(2):161-71. doi:10.3233/NPM-1867.

10. De Lorenzo RA, Morris MJ, Williams JB, et al. Does a simple bedside sonographic measurement of the inferior vena cava correlate to central venous pressure? J Emerg Med. 2012;42(4):429-36. doi:10.1016/j.jemermed.2011.05.082.

11. Zhu Weihua W, Linjun W, Xiaohong, et al. Measurement of brachial artery velocity variation and inferior vena cava variability to estimate fluid responsiveness. Chin Crit Care Med. 2016;28(8):713-7. DOI:10.3760/cma.j.issn.2095-4352.2016.08.009.

12. Wilkman E, Kuitunen A, Pettilä V, Varpula M. Fluid responsiveness predicted by elevation of PEEP in patients with septic shock. Acta Anaesthesiol Scand. 2014;58(1):27-35. doi:10.1111/aas.12229.

13. Mugloo MM, Malik S, Akhtar R. Echocardiographic Inferior Vena Cava Measurement As An Alternative to Central Venous Pressure Measurement in Neonates[J]. Indian J Pediatr. 2017;84(10):751-6.

14. Nagdev AD, Merchant RC, Tirado-Gonzalez A, Sisson CA. Murphy MC.Emergency department bedside ultrasonographic measurement of the caval index for noninvasive determination of low central venous pressure[J]. Ann Emerg Med. 2010;55:290-5.

15. Thanakitcharu P, Charoenwut M, Siriwiwatanakul N. Inferior vena cava diameter and collapsibility index: a practical non-invasive evaluation of intravascular fluid volume in critically-ill patients[J]. J Med Assoc Thail. 2013;96:14-22.

16. Marik PE, Cavallazzi R. Does the central venous pressure predict fluid responsiveness? An updated meta-analysis and a plea for some common sense. Crit Care Med. 2013;41(7):1774-81. doi:10.1097/CCM.0b013e31828a25fd.

17. Cecconi M, De Backer D, Antonelli M, et al. Consensus on circulatory shock and hemodynamic monitoring. Task force of the European Society of Intensive Care Medicine. Intensive Care Med. 2014;40(12):1795-815. doi:10.1007/s00134-014-3525-z]].

18. May CN, Calzavacca $P$, Ishikawa K, et al. Novel targets for sepsis-induced kidney injury: the glomerular arterioles and the sympathetic nervous system. Exp Physiol. 2012;97(11):1168-77. doi:10.1113/expphysiol.2011.061804.

19. Miller LE, Stoller JZ, Fraga MV. Point-of-care ultrasound in the neonatal ICU. Curr Opin Pediatr. 2020;32(2):216-27. doi:10.1097/MOP.0000000000000863.

\section{Tables}


Table 1

Basic Demographic Data

\begin{tabular}{|llll|}
\hline & Control Group & Experimental Group & P \\
\hline Gestational Age (Weeks) & $30.2 \pm 2.7$ & $30.5 \pm 2.2$ & 0.630 \\
\hline Birth Weight (Kg) & $1.28 \pm 0.23$ & $1.29 \pm 0.38$ & 0.911 \\
Male & $12(54.5)$ & $12(50.0)$ & 0.905 \\
\hline Onset of PH (Hours) & $30.6 \pm 10.5$ & $31.0 \pm 10.2$ & 0.877 \\
\hline
\end{tabular}

Table 2

Major Arterial Blood Gas Index at the Onset of PH

\begin{tabular}{|lllllll|}
\hline Group & $\mathrm{pH}$ & $\mathrm{PaCO}_{2}(\mathrm{mmHg})$ & $\mathrm{PaO}_{2}(\mathrm{mmHg})$ & $\begin{array}{l}\text { Lactate } \\
(\mathrm{mmol} / \mathrm{L})\end{array}$ & Ol & $\begin{array}{l}\mathrm{HR} \\
(\mathbf{b p m})\end{array}$ \\
\hline Control & $7.095 \pm 0.103$ & $72.7 \pm 6.3$ & $42.7 \pm 6.3$ & $8.8 \pm 2.3$ & $20.8 \pm 7.8$ & $171 \pm 11$ \\
Experimental & $7.096 \pm 0.105$ & $73.3 \pm 6.6$ & $41.0 \pm 4.5$ & $9.1 \pm 2.5$ & $21.0 \pm 7.6$ & $180 \pm 11$ \\
$\mathrm{P}$ & 0.976 & 0.738 & 0.320 & $0 . .265$ & 0.947 & 0.163 \\
\hline
\end{tabular}


Table 3

Correlation between IVC-Cl and Changes of Plasma $\mathrm{Hb}$ Concentration

\begin{tabular}{|c|lll|}
\hline $\mathbf{H b}$ & $\mathbf{H b}$ & Changes of $\mathrm{Hb}$ & $\mathbf{I V C}-\mathbf{C l}$ \\
\hline 140 & 120 & $14.29 \%$ & 0.5 \\
\hline 150 & 130 & $13.33 \%$ & 0.47 \\
\hline 140 & 100 & $28.57 \%$ & 0.6 \\
\hline 180 & 90 & $50.00 \%$ & 0.65 \\
\hline 175 & 80 & $54.29 \%$ & 0.7 \\
\hline 165 & 120 & $27.27 \%$ & 0.58 \\
\hline 170 & 123 & $27.65 \%$ & 0.55 \\
\hline 167 & 117 & $29.94 \%$ & 0.6 \\
\hline 170 & 100 & $41.18 \%$ & 0.65 \\
\hline 163 & 125 & $23.31 \%$ & 0.61 \\
\hline 157 & 127 & $19.11 \%$ & 0.54 \\
\hline 169 & 128 & $24.26 \%$ & 0.58 \\
\hline 157 & 113 & $28.03 \%$ & 0.6 \\
\hline 155 & 100 & $35.48 \%$ & 0.63 \\
\hline 149 & 105 & $29.53 \%$ & 0.61 \\
\hline 156 & 123 & $21.15 \%$ & 0.55 \\
\hline 139 & 103 & $25.90 \%$ & 0.53 \\
\hline 142 & 114 & $19.72 \%$ & 0.53 \\
\hline 158 & 120 & $24.05 \%$ & 0.53 \\
\hline 167 & 133 & $20.36 \%$ & 0.53 \\
\hline 164 & 117 & $28.66 \%$ & 0.58 \\
\hline 171 & 130 & $23.98 \%$ & 0.57 \\
\hline & & & \\
\hline
\end{tabular}


Table 4

Intake Fluid Volume of Different Groups

\begin{tabular}{|llll|}
\hline Group & $\mathbf{0 - 6 h}(\mathrm{ml} / \mathrm{kg})$ & $\mathbf{6 - 2 4 h}(\mathrm{ml} / \mathrm{kg})$ & $\mathbf{2 4 - 4 8 h}(\mathrm{ml} / \mathrm{kg})$ \\
\hline Control & $60 \pm 4$ & $93 \pm 4$ & $102 \pm 10$ \\
\hline Experimental & $64 \pm 6$ & $71 \pm 8$ & $105 \pm 9$ \\
\hline P & 0.013 & 0.000 & 0.361 \\
\hline
\end{tabular}

Due to technical limitations, table 5 is only available as a download in the Supplemental Files section.

\section{Figures}

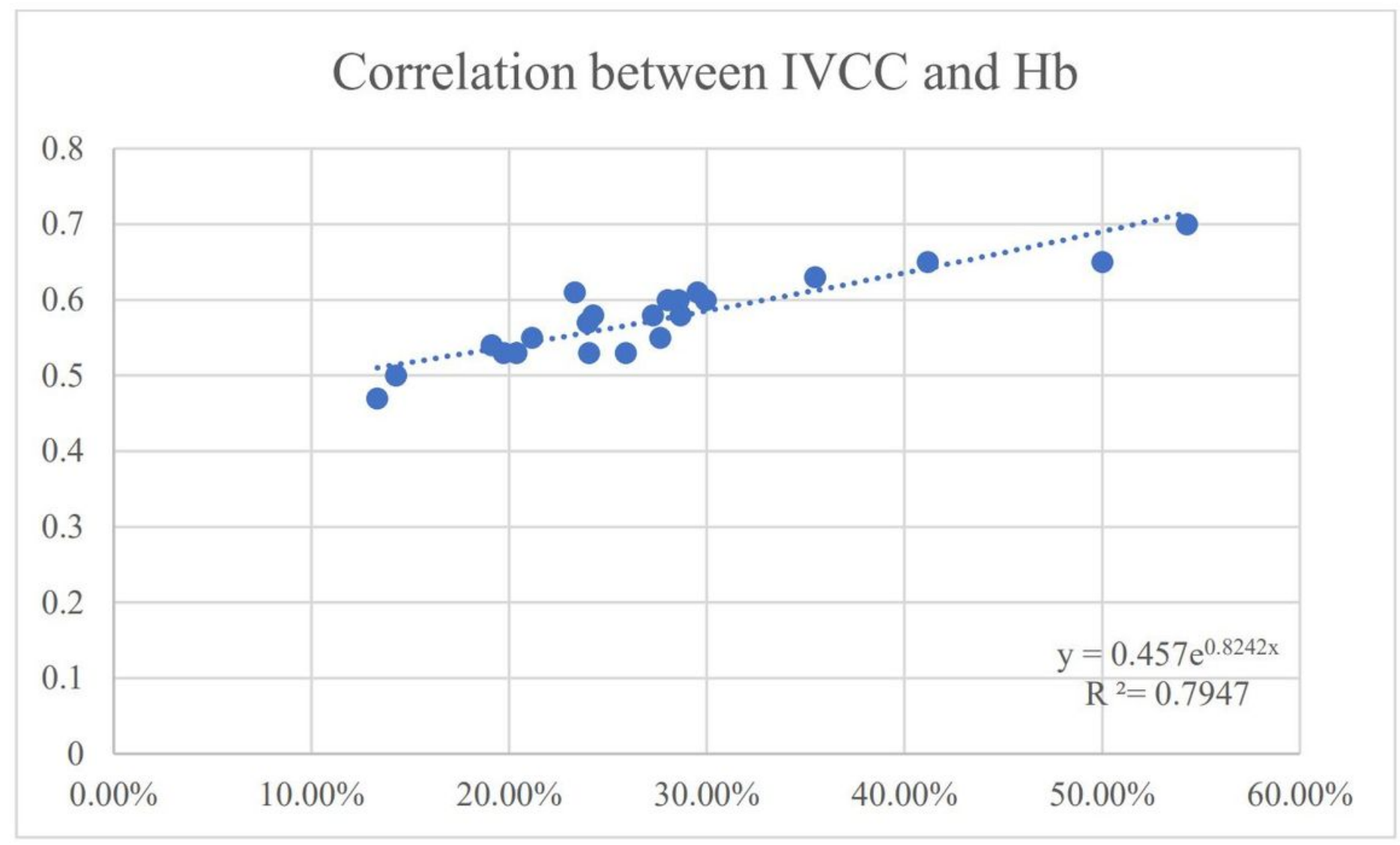

Figure 1

Linear relation between IVC-Cl and $\mathrm{Hb}$ (data from Table 3)

\section{Supplementary Files}

This is a list of supplementary files associated with this preprint. Click to download. 
- Table5.jpg

Page $13 / 13$ 